\title{
Bulk locality in the AdS/CFT correspondence
}

\author{
Seiji Terashima* \\ Yukawa Institute for Theoretical Physics, Kyoto University, Kyoto 606-8502, Japan
}

(Received 26 April 2021; accepted 2 September 2021; published 6 October 2021)

\begin{abstract}
In this paper, we study the bulk local states in the AdS/CFT correspondence in the large $N$ limit using the formula explicitly relating the bulk local operators and the conformal field theory (CFT) local operators. We identify the bulk local state in terms of CFT local states and find that the bulk local state corresponds to a CFT state supported in the whole space, which means a version of the subregion duality is not valid. On the other hand, CFT states supported in a space region are expressed in terms of the bulk states supported in a certain region. We find that the quantum error correction proposal is not realized although the puzzles of the radial locality which motivated the proposal are resolved in our analysis. For the understating of the bulk local states, an explicit realization of an analog of the Reeh-Schlieder theorem plays an important role.
\end{abstract}

DOI: 10.1103/PhysRevD.104.086014

\section{INTRODUCTION AND SUMMARY}

The AdS/CFT correspondence [1] is highly nontrivial and important in various aspects of physics and has been investigated intensively. There is no proof for this correspondence, thus it is still a conjecture although there are many evidences.

The key ingredient of the AdS/CFT correspondence is the locality in the bulk, which is needed for the emergence of the extra dimension (for a recent discussion on the bulk locality, see [2,3]). In particular, the local states in the bulk should be understood from the states of $\mathrm{CFT}_{d}$. In order to study the correspondence between the bulk theory and the conformal field theory (CFT), some bulk-boundary correspondence, like the Gubser-Klebanov-Polyakov-Witten (GKPW) relation [4,5] or the Banks-Douglas-HorowitzMartinec (BDHM) relation [6] is usually assumed. Instead of assuming such bulk-boundary correspondences, with the natural assumptions on the large $N$ strong coupling $\mathrm{CFT}_{d}$, we can show the equivalence between the spectrum (i.e., the states and the Hamiltonian) of the $\mathrm{CFT}_{d}$ and the spectrum of the bulk theory on the $(d+1)$-dimensional anti-de Sitter space ( $\left.\operatorname{AdS}_{d+1}\right)$ in the (naive) large $N$ limit, in which the bulk gravity theory will become a free theory [7]. ${ }^{1}$ Here, the Hamiltonian is given by the dilatation operator. Then, the bulk local operator and the bulk local

\footnotetext{
*terasima@yukawa.kyoto-u.ac.jp

${ }^{1}$ Here, we considered the (asymptotically) global $\mathrm{AdS}_{d+1}$ only, because the spectrum of the theory will be continuous and ambiguous for the Poincaré patch of AdS.

Published by the American Physical Society under the terms of the Creative Commons Attribution 4.0 International license. Further distribution of this work must maintain attribution to the author(s) and the published article's title, journal citation, and DOI. Funded by SCOAP ${ }^{3}$.
}

state are explicitly given by the $\mathrm{CFT}_{d}$ operator and the $\mathrm{CFT}_{d}$ state, using the explicit identification of the energy eigenstates in $\mathrm{CFT}_{d}$ to the those in the bulk theory on $\operatorname{AdS}_{d+1}{ }^{2}{ }^{2}$ Furthermore, it was shown that the GKPW and BDHM relations are consequences of this identification.

Note that these results in [7] are based on some earlier works [6,8,9] (see also [10-28]) and the identification in [7] may be consistent with the Hamilton-Kabat-LifschytzLowe (HKLL) reconstruction [16] in which assuming the BDHM relation [6] which relates the $\mathrm{CFT}_{d}$ primary field as a limit of bulk field to the boundary, the bulk local operator is reconstructed from the $\mathrm{CFT}_{d}$ operator. Moreover, the bulk local state at the center of a time slice of $\mathrm{AdS}_{d+1}$ is also explicitly reconstructed by imposing the invariance under some "rotations" symmetries of $\mathrm{AdS}_{d+1}$ which are expected to fix the center [11-13]. The results in [7] are consistent with these also. ${ }^{3}$

In [7], the bulk locality in the large $N$ limit comes from the large $N$ factorization, which guarantees the theory is (generalized) free and the completeness condition of the spectrum, which gives the Fock space together with the conformal symmetry, which may guarantee the Lorentz symmetry for the local neighborhood of a spacetime point. On the other hand, in [29] some problems or puzzles related to the locality in the radial direction in the bulk were given,

\footnotetext{
${ }^{2} \mathrm{~A}$ local state in this paper means a state obtained by acting a local operator on the vacuum. More precisely, the local operators and states should be smeared over a region in the spacetime.

${ }^{3}$ For a state at the center, we can check that the construction in [7] gives same state given by [11-13]. However, there are states which are invariant under the "rotations" symmetries, but is not a local state [7]. In [26], the bulk local states not at the center are constructed using the conformal transformation also for $\mathrm{CFT}_{2}$. These could be different from the ones we will use in this paper because the local operator with spacetime derivatives is a local operator.
} 
and the quantum error correction codes are claimed to be the key to solve these problems (for a review, see [30]). However, the structure of the quantum error correction codes has not been explicitly identified in realistic models of the AdS/CFT correspondence. Thus, it is worth investigating these puzzles in the bulk local states or the bulk local operator expressed by the energy eigenstates of $\mathrm{CFT}_{d}$ in the large $N$ limit using the results in [7].

In this paper, we show how these puzzles of the radial locality are solved in terms of the bulk and CFT local operators or states which are given by the energy eigenstates of $\mathrm{CFT}_{d}$. ${ }^{4}$ The resolution is partly based on the fact that we need the "cutoff" for the high energy states in the low-energy effective theory which is regarded as the bulk (gravity) theory. Note that this cutoff is also crucial for the error correction code proposal [29] although this proposal is completely irrelevant for the analysis in this paper. Indeed, it is about low energy states (or code subspace), thus it is obvious that the cutoff is needed, even if we consider the large $\mathrm{N}$ limit. In other words, in the formal large $N$ expansion, we can not resolve the puzzles. We need the large, but finite $N$ effects (i.e., finite Planck length effects) for the resolution of them.

With this cutoff we explicitly identify the bulk local state in terms of CFT local states. We also explicitly identify CFT states supported in a space region in terms of the bulk local states. We find that the bulk local state corresponds to a CFT state supported in the whole space $S^{d-1}$, not a subregion. This means that the subregion duality [31-35] is not valid in our model. ${ }^{5}$ On the other hand, we argue that a CFT state supported in a space region $A$ corresponds to a a bulk state supported in a certain space region. We call this bulk region as the minimal surface wedge of $A$, which is a kind of generalization of the entanglement wedge. Furthermore, we find that two bulk local states at a same spacetime point constructed from CFT states supported in space regions $A$ and $A^{\prime}$ are different generically if $A \neq A^{\prime}$, even in the low energy theory. This means that the quantum error correction code proposal [29] is not realized although the puzzles of the radial locality raised in [29] are resolved.

We stress that the model considered in this paper is the generalized free theory which is the universal low-energy theory of AdS/CFT, although we consider only the scalar. Namely, we study the holographic CFT itself, not a model of the holographic CFT.

\footnotetext{
${ }^{4}$ In this paper, we only consider the bulk (free) scalar field and the corresponding sector of CFT in the naive large $N$ limit, for simplicity.

${ }^{5}$ In this paper, the subregion duality means that the operators supported in the CFT subregion $A$ is equivalent to the operators supported in a corresponding bulk subregion $a$. Another weaker version of it is that the CFT density matrix for the region $A$, which is defined by the tracing out, is equivalent to the density matrix for the bulk theory defined in a bulk region $a$ with an appropriate boundary condition. This will be consistent with our results.
}

One of the important topics in this paper is the ReehSchlieder theorem. We explicitly construct a CFT local state at a spacetime point which is effectively equivalent to a CFT local state at a different spacetime point. Hence, it is important to find which state is effectively local with the cutoff we introduced and then we call them the CFT effective local states at each spacetime point. The CFT effective local states on the time slice $t=0$ do not span even the low-energy states. However, by the time evolution, a CFT effective local state at $t=t_{0} \neq 0$ gives a CFT state supported in a region at $t=0$, which is not given by effective local states on $t=0$. If we consider the CFT effective local states in whole spacetime (or the spacetime region $\pi \leq t \leq \pi$ ) and consider the time evolution of them to the time slice $t=0$, then any low energy state is expected to be given by them because in the low-energy theory the effective local states play the role of the local states. ${ }^{6}$ We will see that the maximum distance from the boundary in radial direction $z$ of the bulk local state at $t=0$ constructed from such a CFT effective local state at $t=t_{0}$ is $\left|t_{0}\right|$ because of the bulk causality.

In this paper, we concentrate on the free field limit of the bulk theory around the AdS spacetime. A generalization of [7] to general classical backgrounds was done in [36] and showed that the Einstein equation was derived with assumptions made in [7]. It is interesting to investigate how the analysis in the paper can be generalized to a general background. In particular, for the black hole, we expect that the brick wall [37] will appear [38] instead of the horizon and it is interesting to study how the locality near the brick wall is modified.

This paper is organized as follows. In the next section we review the relation between the free scalar field on $\operatorname{AdS}_{d+1}$ and the large $N \mathrm{CFT}_{d}$ according to [7]. Based on this, we will describe the bulk local states and the CFT local states in terms of the energy eigenstates. In Sec. III we study the bulk locality in AdS/CFT correspondence in the naive large $N$ limit. We explicitly identify the bulk local state as a CFT state and we also identify a CFT state supported in a space region as a bulk state.

\section{BULK LOCAL STATES AND CFT LOCAL STATES}

In this section, we will review the relation between free scalar field on $\mathrm{AdS}_{d+1}$ and large $N \mathrm{CFT}_{d}$ according to [7]. Based on this, we will describe the bulk local states and the CFT local states in terms of the energy eigenstates. We will consider the low-energy theory with the energy cutoff $\omega_{c}$, which is related to the Planck mass, in order to obtain the

\footnotetext{
${ }^{6}$ Note that any CFT state is given by a superposition of the CFT local states in the $t=0$ time slice of spacetime. For a small number of (free) fields, any state (without a cutoff) will be given by a effectively local states in the time slice, which implies that there is no bulk dual for such a CFT.
} 
local bulk theory picture. This cutoff also introduces the truncation of the states for sufficiently high energy states. For the local operator and the local states, this cutoff gives the effective smearing over the time with the length scale $1 / \omega_{c}$. Below, we will also explain such descriptions of the local states in the low-energy theory.

\section{A. Relation between free scalar field on $\operatorname{AdS}_{d+1}$ and large $N$ CFT $_{d}$}

In this subsection, we will review local operators and states of the (free) scalar field on $\mathrm{AdS}_{d+1}$ in terms of $\mathrm{CFT}_{d}$ in the large $N$ limit, following [7].

Let us consider the free scalar field for which the action is given by

$$
S_{\text {scalar }}=\int d^{d+1} x \sqrt{-\operatorname{det}(g)}\left(\frac{1}{2} g^{M N} \nabla_{M} \phi \nabla_{N} \phi+\frac{m^{2}}{2} \phi^{2}\right),
$$

where $M, N=1, \ldots, d+1$, on global $\operatorname{AdS}_{d+1}$. The $\operatorname{AdS}_{d+1}$ metric is

$$
d s_{\mathrm{AdS}}^{2}=-\left(1+r^{2}\right) d t^{2}+\frac{1}{1+r^{2}} d r^{2}+r^{2} d \Omega_{d-1}^{2},
$$

where $0 \leq r<\infty,-\infty<t<\infty$, and $d \Omega_{d-1}^{2}$ is the metric for the $d$-1-dimensional round unit sphere $S^{d-1}$. We set the AdS scale $l_{\mathrm{AdS}}=1$ in this paper. By the coordinate change $r=\tan \rho$, the metric is also written as

$$
d s_{\mathrm{AdS}}^{2}=\frac{1}{\cos ^{2}(\rho)}\left(-d t^{2}+d \rho^{2}+\sin ^{2}(\rho) d \Omega_{d-1}^{2}\right),
$$

where $0 \leq \rho<\pi / 2$. With $z=\pi / 2-\rho$, the metric is given by

$$
d s_{\text {AdS }}^{2}=\frac{1}{\sin ^{2}(z)}\left(-d t^{2}+d z^{2}+\cos ^{2}(z) d \Omega_{d-1}^{2}\right) .
$$

The boundary of the $\operatorname{AdS}_{d+1}$ is located at $\rho=\pi / 2$ or $z=0$.

We expand the quantized field $\hat{\phi}$ with the spherical harmonics $Y_{l m}(\Omega)$,

$$
\hat{\phi}(t, \rho, \Omega)=\sum_{n, l, m}\left(\hat{a}_{n l m}^{\dagger} e^{i \omega_{n} t}+\hat{a}_{n l m} e^{-i \omega_{n} t}\right) \psi_{n l m}(\rho) Y_{l m}(\Omega),
$$

where $\Omega$ represents the coordinates of $S^{d-1}$ and

$$
\begin{aligned}
\psi_{n l}(\rho)= & \frac{1}{N_{n l}} \sin ^{l}(\rho) \cos ^{\Delta}(\rho) \\
& \times{ }_{2} F_{1}\left(-n, \Delta+l+n, l+\frac{d}{2}, \sin ^{2}(\rho)\right),
\end{aligned}
$$

where $\Delta$ is given by $\Delta=d / 2 \pm \sqrt{m^{2}+d^{2} / 4}$, and

$$
\omega_{n l}=2 n+l+\Delta,
$$

where $n, l=0,1,2,3, \ldots$. The normalization constant is given by

$$
N_{n l}=(-1)^{n} \sqrt{\frac{n ! \Gamma\left(l+\frac{d}{2}\right)^{2} \Gamma\left(\Delta+n+1-\frac{d}{2}\right)}{\Gamma\left(n+l+\frac{d}{2}\right) \Gamma(\Delta+n+l)}} .
$$

The operators $\hat{a}_{n l m}$ satisfy the commutation relation

$$
\left[\hat{a}_{n l m}, \hat{a}_{n^{\prime} l^{\prime} m^{\prime}}^{\dagger}\right]=\delta_{n, n^{\prime}} \delta_{l, l^{\prime}} \delta_{m, m^{\prime}},
$$

and the Hamiltonian is such that

$$
\left[\hat{H}, \hat{a}_{n l m}\right]=-\omega_{n l} .
$$

The Hilbert space is the Fock space spanned by $\prod_{n, l, m}\left(\hat{a}_{n l m}^{\dagger}\right)^{\mathcal{N}_{n l m}}|0\rangle$, where $\mathcal{N}_{n l m}$ is a non-negative integer. We choose the constant shift of the Hamiltonian as $\hat{H}|0\rangle=0$ where $|0\rangle$ is the vacuum, i.e., $\hat{a}_{n l m}|0\rangle=0$.

Now, let us consider a scalar primary field $\mathcal{O}_{\Delta}(x)$ in $\mathrm{CFT}_{d}$ on $\mathbf{R} \times S^{d-1}$ where $\mathbf{R}$ is the time direction and the radius of $S^{d-1}$ is taken to be 1 . (For a review of the $\mathrm{CFT}_{d}$, see for example, [39-41].) Any state in CFT can be obtained as a linear combination of the primary state $|\Delta\rangle=$ $\lim _{x \rightarrow 0} \mathcal{O}_{\Delta}(x)|0\rangle$ with the $\hat{P}^{\mu}$, for example,

$$
\hat{P}^{\mu_{1}} \hat{P}^{\mu_{2}} \cdots \hat{P}^{\mu_{l}}|\Delta\rangle .
$$

We also define the operator

$$
\hat{\mathcal{O}}_{\Delta}=\lim _{x \rightarrow 0} \hat{\mathcal{O}}_{\Delta}^{+}(x),
$$

where $\hat{\mathcal{O}}_{\Delta}^{+}(x)$ is the regular part of $\mathcal{O}_{\Delta}(x)$ in $x^{\mu} \rightarrow 0$ limit $^{7}$ which can be expanded by the polynomial of $x^{\mu}{ }^{8}$ This satisfies $|\Delta\rangle=\hat{\mathcal{O}}_{\Delta}|0\rangle$ where $|0\rangle$ is the conformal vacuum. It was shown in [7] that the spectrum of this $\mathrm{CFT}_{d}$ in the large $N$ limit is equivalent to the spectrum of free scalar on $\mathrm{AdS}_{d+1}$ under some natural assumptions on the spectrum. The identification of the CFT states to the states of the Fock space of the scalar fields in AdS is explicitly given by the identification of the raising operators as

$$
\hat{a}_{n l m}^{\dagger}=c_{n l} s_{(l, m)}^{\mu_{1} \mu_{2} \ldots \mu_{l}} P_{\mu_{1}} P_{\mu_{2}} \cdots P_{\mu_{l}}\left(P^{2}\right)^{n} \hat{\mathcal{O}}_{\Delta},
$$

\footnotetext{
${ }^{7}$ This should be done after taking the large $N$ limit. More precisely, $\hat{\mathcal{O}}_{\Delta}$ is the sum of the operators of dimension $\Delta$ up to $1 / N$ corrections in $\mathcal{O}_{\Delta}(x)$.

${ }^{8}$ For the stress energy tensor in two dimensional theory, $\hat{\mathcal{O}}_{\Delta}$ is $L_{-2}$ or $\tilde{L}_{-2}$.
} 
where $c_{n l}$ is the normalization constant, which was determined in [7], $P^{\mu}$ acts on an operator such that $P^{\mu} \hat{\phi}=\left[\hat{P}^{\mu}, \hat{\phi}\right]$, and $s_{(l, m)}^{\mu_{1} \mu_{2} \ldots \mu_{l}}$ is a normalized rank $l$ symmetric traceless constant tensor.

\section{B. Bulk local states}

We will decompose the local operator in the bulk description to positive and negative frequency modes as

$$
\hat{\phi}(t, \rho, \Omega)=\hat{\phi}^{+}(t, \rho, \Omega)+\hat{\phi}^{-}(t, \rho, \Omega),
$$

where $\hat{\phi}^{-}(t, \rho, \Omega)=\left(\hat{\phi}^{+}(t, \rho, \Omega)\right)^{\dagger}$. In this paper, we will concentrate on the one particle states in the naive large $N$ limit only. For this, the commutator of the operators $\hat{\phi}(\rho, \Omega) \equiv \hat{\phi}(t=0, \rho, \Omega)$ is a constant and given by the overlap of the corresponding states, $\hat{\phi}^{+}(\rho, \Omega)|0\rangle$, which is

$$
\begin{aligned}
& \left\langle 0\left|\left[\hat{\phi}_{1}(\rho, \Omega), \hat{\phi}_{2}\left(\rho^{\prime}, \Omega^{\prime}\right)\right]\right| 0\right\rangle \\
& \quad=\left\langle 0\left|\hat{\phi}_{1}^{-}(\rho, \Omega) \hat{\phi}_{2}^{+}\left(\rho^{\prime}, \Omega^{\prime}\right)\right| 0\right\rangle-\left\langle 0\left|\hat{\phi}_{2}^{-}\left(\rho^{\prime}, \Omega^{\prime}\right) \hat{\phi}_{1}^{+}(\rho, \Omega)\right| 0\right\rangle \\
& \quad=2 i \operatorname{Im}\left(\left\langle 0\left|\hat{\phi}_{1}^{-}(\rho, \Omega) \hat{\phi}_{2}^{+}\left(\rho^{\prime}, \Omega^{\prime}\right)\right| 0\right\rangle\right),
\end{aligned}
$$

where $\hat{\phi}_{i}$ represents $\hat{\phi}$ with some spacetime derivatives and $\hat{\phi}_{i}^{ \pm}$is the positive/negative frequency modes of $\hat{\phi}_{i}$. Thus, we will study the states, instead of the operators in order to understand the locality in the bulk. In particular, the commutator of the operators is zero if the corresponding overlap vanishes.

Using (2.13), the bulk local operator is expressed as

$$
\begin{aligned}
\hat{\phi}^{+} & t=0, \rho, \Omega) \\
& =\sum_{n, l, m} \psi_{n l}(\rho) Y_{l m}(\Omega) \hat{a}_{n l m}^{\dagger} \\
& =\sum_{n, l, m} \psi_{n l}(\rho) Y_{l m}(\Omega) c_{n l} s_{(l, m)}^{\mu_{1} \mu_{2} \ldots \mu_{l}} P_{\mu_{1}} P_{\mu_{2}} \cdots P_{\mu_{l}}\left(P^{2}\right)^{n} \hat{\mathcal{O}}_{\Delta},
\end{aligned}
$$

where only the CFT operators appear in the last line. ${ }^{9}$ The wave function for the radial direction can be rewritten as

$\psi_{n l}(\rho)=\frac{1}{\mathcal{N}_{n l}} \sin ^{l}(\rho) \cos ^{\Delta}(\rho) P_{n}^{l+d / 2-1, \Delta-d / 2}(\cos (2 \rho))$,

where $P_{n}^{\alpha, \beta}(x)$ is the Jacobi polynomial defined by

\footnotetext{
${ }^{9}$ The derivatives of a local operator with $t, \rho, \Omega$ is also a local operator, for example, the momentum $\hat{\pi}(t, \rho, \Omega)=$ $\sqrt{-g} g^{t t} \frac{\partial}{\partial t} \hat{\phi}(t, \rho, \Omega)$ is a local operator.
}

$$
\begin{aligned}
P_{n}^{\alpha, \beta}(x)= & \frac{(-1)^{n}}{2^{n} n !}(1-x)^{-\alpha}(1+x)^{-\beta} \\
& \times \frac{d^{n}}{d x^{n}}\left((1-x)^{\alpha}(1+x)^{\beta}\left(1-x^{2}\right)^{n}\right),
\end{aligned}
$$

and the normalization constant $\mathcal{N}_{n l}$ is given by

$$
\mathcal{N}_{n l}=(-1)^{n} \sqrt{\frac{\Gamma\left(n+l+\frac{d}{2}\right) \Gamma\left(n+1+\Delta-\frac{d}{2}\right)}{\Gamma(n+l+\Delta) \Gamma(n+1)}} .
$$

For later use, we will compute the large $n, l$ limit of $\mathcal{N}_{n l}$ using the following formula; $\lim _{z \rightarrow \infty} \Gamma(z+a) / \Gamma(z)=z^{a}$. For $l \rightarrow \infty$ with a fixed $n$, we have $(-1)^{n} \mathcal{N}_{n l} \rightarrow$ $\sqrt{\frac{\Gamma\left(n+1+\Delta-\frac{d}{2}\right)}{\Gamma(n+1)}} \frac{1}{l^{\Delta / 2-d / 4}}$. For $n \rightarrow \infty$ with a fixed $l$, $(-1)^{n} \mathcal{N}_{n l} \rightarrow 1$. For $n \rightarrow \infty$ with a fixed $l / n,(-1)^{n} \mathcal{N}_{n l} \rightarrow$ $\left(\frac{n}{n+l}\right)^{\Delta / 2-d / 4}$. Note that these limiting values are not exponential, but powers of $n, l$.

Then, the bulk local state at $\rho=\rho_{0}, \Omega=\Omega_{0}$ is given by

$$
\begin{aligned}
\left|\rho_{0}, \Omega_{0}, t\right\rangle & \equiv \hat{\phi}\left(t, \rho_{0}, \Omega_{0}\right)|0\rangle \\
& =\sum_{n, l, m} e^{i t(2 n+l+\Delta)} \psi_{n l}\left(\rho_{0}\right) Y_{l m}\left(\Omega_{0}\right) \hat{a}_{n l m}^{\dagger}|0\rangle
\end{aligned}
$$

The other bulk local operators and the bulk local states are obtained from these by applying a finite number of spacetime derivatives. ${ }^{10}$

The overlap between $\hat{\phi}\left(t=0, \rho=\rho_{1}, \Omega=\Omega_{1}\right)|0\rangle$ and $\hat{\pi}\left(t=0, \rho=\rho_{0}, \Omega=\Omega_{0}\right)|0\rangle$, where $\hat{\pi}(t, \rho, \Omega)=$ $\sqrt{-g} g^{t t} \frac{\partial}{\partial t} \hat{\phi}(t, \rho, \Omega)=\left(\frac{\sin \rho}{\cos \rho}\right)^{d-1} \frac{\partial}{\partial t} \hat{\phi}(t, \rho, \Omega)$ is the momentum which satisfies $\left[\hat{\phi}\left(t=0, \rho=\rho_{1}, \Omega=\Omega_{1}\right)\right.$, $\left.\hat{\pi}\left(t=0, \rho=\rho_{0}, \Omega=\Omega_{0}\right)\right]=i \delta\left(\rho_{0}-\rho_{1}\right) \delta\left(\Omega_{0}-\Omega_{1}\right)$, can be computed from

$$
\begin{aligned}
\left.\left(\left\langle\rho_{1}, \Omega_{1}, t\left|\frac{\partial}{\partial t}\right| \rho_{0}, \Omega_{0}, t\right\rangle\right)\right|_{t=0} & \\
= & \sum_{l, m}\left(\sum_{n} i(2 n+l+\Delta) \psi_{n l}\left(\rho_{1}\right) \psi_{n l}\left(\rho_{0}\right)\right) \\
& \times Y_{l m}\left(\Omega_{1}\right) Y_{l m}\left(\Omega_{0}\right),
\end{aligned}
$$

which is (formally) proportional to $i \delta\left(\rho_{0}-\rho_{1}\right) \delta\left(\Omega_{0}-\Omega_{1}\right)$ because of the orthogonality properties of the Jacobi polynomials and the hyperspherical harmonics. We will denote the bulk local state at $\rho=\rho_{0}, \Omega=\Omega_{0}$ on the $t=0$ slice simply as

\footnotetext{
${ }^{10}$ In the approximation where bulk theory is free, the time derivative does not produce an independent local field by the equations of motion, except the momentum. The products and the linear combinations of the local operators are also local operators, however, we will not consider the products for simplicity.
} 


$$
\left|\rho_{0}, \Omega_{0}\right\rangle \equiv \sum_{n, l, m} \psi_{n l}\left(\rho_{0}\right) Y_{l m}\left(\Omega_{0}\right) \hat{a}_{n l m}^{\dagger}|0\rangle
$$

The overlap of these, $\left\langle\rho_{1}, \Omega_{1} \mid \rho_{0}, \Omega_{0}\right\rangle$, may vanish (formally) except $\rho_{0}=\rho_{1}$ and $\Omega_{0}=\Omega_{1}$.

Of course, these local states (and corresponding local operators) themselves are not well defined. Indeed, they have a divergent energy, which means these are ill defined, and need to be smeared or regularized, for example, by integrating $\rho, \Omega$ with the Gaussian factors of the center at $\rho_{0}, \Omega_{0}{ }^{11}$ Other ways of regularization are by inserting some factors which decay exponentially for large $n, l$, for example, $e^{-\hat{H} / \omega_{c}}$. Effectively both of these will introduce the cutoff of the contributions for the large $n, l$ in the summations.

Note that if we consider the low-energy effective theory with a cutoff of energy $\omega_{c}$, there are no local operators smeared over the region smaller than $1 / \omega_{c}$ and the local operator should be smeared over a region of size of at least $1 / \omega_{c}$. This means that there is the largest energy cutoff, around and above where the bulk locality is meaningless in the low-energy effective theory. In the AdS/CFT correspondence, the bulk description should be the low-energy effective theory. This is because the complete independence assumed in [7] should be violated for the finite $N$ CFT and the multiparticle states, which are related to the multitrace operators, with a large energy are not independent from the states with lower numbers of particles. Then, above this energy scale the gravity picture (or the bulk locality) is not valid. This invalidity of the gravity picture has been known as the stringy exclusion principle [42], at least, for some special examples of $\mathrm{AdS}_{3} / \mathrm{CFT}_{2}$. The largest cutoff $\omega_{c}$ is unknown for general models, however, it is expected to be $\omega_{c}<\mathcal{O}\left(N^{2}\right)$ because the degrees of freedom of the CFT is $\mathcal{O}\left(N^{2}\right)$. Therefore, in this paper we consider the bulk locality and the bulk local operators with the cut-off $\omega_{c}=$ $\mathcal{O}\left(N^{2}\right)$ although we will only use the fact that a cutoff of the energy is needed and we will consider the leading order in $1 / \omega_{c}$ expansion. $^{12}$

\footnotetext{
${ }^{11}$ We can also regularize this by integrating over the time with, for example, the Gaussian factor. The regularization with the smooth function supported on a spacetime region is better for the notion of the local operators in the axiomatic quantum field theory. We will not use such regularization because it is technically difficult for the actual computations and what we will consider below is the low-energy effective field theory which is not described by the axiomatic quantum field theory.

${ }^{12}$ The gravity picture will be not valid even below this energy scale, for example, the cutoff will be the plank scale, $\mathcal{O}\left(N^{2 \frac{1}{d-1}}\right)$. The energy of the local operator will be proportional to the cutoff energy and if the energy of the operator is proportional to the plank scale, the back reaction to the geometry, which could induces the topology change, should be incorporated. This backreaction may change the notion of the bulk locality. Thus, the cutoff is expected to be much less than the Planck scale.
}

With the cutoff, the overlap of two different bulk local states (2.22),

$$
\begin{aligned}
\left\langle\rho_{1}, \Omega_{1} \mid \rho_{0}, \Omega_{0}\right\rangle= & \sum_{l, m}\left(\sum_{n} \psi_{n l}\left(\rho_{1}\right) \psi_{n l}\left(\rho_{0}\right)\right) \\
& \times Y_{l m}\left(\Omega_{1}\right) Y_{l m}\left(\Omega_{0}\right),
\end{aligned}
$$

vanishes, up to $1 / \omega_{c}$ corrections, except $\rho_{0}=\rho_{1}$ and $\Omega_{0}=\Omega_{1}$.

We can consider the local state smeared over angular direction $\Omega$, for example by a Gaussian factor, with length scale $1 / l_{c}$ adding to the energy cutoff $\omega_{c}$. This induces the effective cutoff $l_{c}$ for the summation over $l$ in (2.22). ${ }^{13}$ Let us consider these local states with a regularization satisfies

$$
\omega_{c} \gg l_{c},
$$

which are important for the discussion below. For this case, we can explicitly see how the overlap between the two local states at different space points almost vanishes, as we will see below. The dominant contributions in the summation over $n$ in (2.22) are those for $l_{c} \ll n\left(\leq \omega_{c}\right)$ and the asymptotic behavior of $\psi_{n l}(\rho)$ for large $n$ (with $l$ and $z$ fixed) is computed, using the asymptotic behavior of Jacobi polynomial [43], as

$$
\begin{aligned}
\psi_{n l}(\rho)= & \frac{1}{\sqrt{\pi n}}(\tan z)^{\frac{d-1}{2}} \cos \left((2 n+l+\Delta) z-\frac{\pi}{2}\left(\Delta-\frac{d}{2}+\frac{1}{2}\right)\right) \\
& +\mathcal{O}\left(n^{-3 / 2}\right),
\end{aligned}
$$

where

$$
z=\pi / 2-\rho
$$

and the boundary is at $z=0$. In the expression of the overlap of two different bulk local states,

$$
\begin{aligned}
\left\langle\rho_{1}, \Omega_{1} \mid \rho_{0}, \Omega_{0}\right\rangle= & \sum_{l=0}^{l_{c}}\left(\sum_{n=0}^{\left[\left(\omega_{c}-l-\Delta\right) / 2\right]} \psi_{n l}\left(\rho_{1}\right) \psi_{n l}\left(\rho_{0}\right)\right) \\
& \times \sum_{m} Y_{l m}\left(\Omega_{1}\right) Y_{l m}\left(\Omega_{0}\right),
\end{aligned}
$$

the summation over $n$ is almost canceled if $\rho_{0} \neq \rho_{1}$, and if $\rho_{0}=\rho_{1}$ they are almost $l$-independent, because of the phase factor in (2.25). ${ }^{14}$ Then, for the angular directions, $\sum_{l, m} Y_{l m}\left(\Omega_{1}\right) Y_{l m}\left(\Omega_{0}\right) \sim \delta\left(\Omega_{0}-\Omega_{1}\right)$, where the large $l$ contributions are almost canceled if $\Omega_{0} \neq \Omega_{1}$ because of

\footnotetext{
${ }^{13}$ Because the precise form of the regularization is expected to be irrelevant, we will just regard the regularization as the cutoff $l_{c}$ for the summation over $l$.

${ }^{14}$ The $\frac{1}{\sqrt{n}}$ factor in $(2.25)$ is canceled if we consider the commutator between the bulk local field and its momentum.
} 
phase cancellations. Here, the resolution of $\rho$ is $1 / \omega_{c}$ and the resolution of the angular direction is $1 / l_{c}$.

Note that these local states with the regularization, which satisfies $\omega_{c} \gg l_{c}$, is squeezed in the radial direction and move in the radial direction only; thus among the local states these are a special class of states because of the condition $\omega_{c} \gg l_{c}$.

\section{C. $\mathrm{CFT}_{\boldsymbol{d}}$ local states}

We have considered the bulk local state in $A d S_{d+1}$, i.e, $\phi(t, \rho, \Omega)$. On the other hand, the local state in the $\mathrm{CFT}_{d}$, i.e., $\mathcal{O}_{\Delta}(t, \Omega)|0\rangle$, will be different from the bulk local state. This is possible because the notions of locality are different between the finite $N \mathrm{CFT}_{d}$ and the bulk theory.

We can represent the $\mathrm{CFT}_{d}$ local state $\mathcal{O}_{\Delta}(t, \Omega)|0\rangle$ as a linear combination of the (normalized) energy eigenstates,

$$
c_{n l} s_{(l, m)}^{\mu_{1} \mu_{2} \ldots \mu_{l}} P_{\mu_{1}} P_{\mu_{2}} \cdots P_{\mu_{l}}\left(P^{2}\right)^{n} \hat{\mathcal{O}}_{\Delta}|0\rangle,
$$

which is identified as $\hat{a}_{n l m}^{\dagger}|0\rangle$. This rewriting was explicitly done essentially in [7] by using the hyperspherical Bessel function in order to derive the BDHM [6] extrapolation formula,

$$
\lim _{\rho \rightarrow \pi / 2} \frac{\hat{\phi}(\rho, \Omega)}{\cos ^{\Delta}(\rho)}=\sqrt{\frac{\pi}{2}} \sqrt{\frac{\Gamma(\Delta)}{\Gamma(\Delta+1-d / 2) \Gamma(d / 2)}} \mathcal{O}_{\Delta}(\Omega) .
$$

If we define

$$
\psi_{n l}^{\mathrm{CFT}} \equiv \sqrt{\frac{2}{\pi} \frac{\Gamma(\Delta+1-d / 2) \Gamma(d / 2)}{\Gamma(\Delta)}} \lim _{\rho \rightarrow \pi / 2} \frac{\psi_{n l}(\rho)}{\cos ^{\Delta}(\rho)},
$$

where

$$
\begin{aligned}
\lim _{\rho \rightarrow \pi / 2} \frac{\psi_{n l}(\rho)}{\cos ^{\Delta}(\rho)}= & \sqrt{\frac{\Gamma(n+\Delta+1-d / 2) \Gamma(n+l+\Delta)}{\Gamma(n+1) \Gamma(n+l+d / 2)}} \\
& \times \frac{1}{\Gamma(\Delta+1-d / 2)},
\end{aligned}
$$

the local state in the $\mathrm{CFT}_{d}$ is written as

$$
\mathcal{O}_{\Delta}(\Omega)|0\rangle=\sum_{n, l, m} \psi_{n l}^{\mathrm{CFT}} Y_{l m}(\Omega) \hat{a}_{n l m}^{\dagger}|0\rangle
$$

where we have taken $t=0$. Note that $\psi_{n l}^{\mathrm{CFT}}=\sqrt{\frac{2}{\pi}}$ is a constant for $\Delta=d / 2$.

For later use, we will compute the large $n, l$ limit of $\psi_{n l}^{\mathrm{CFT}}$ up to numerical factors which do not depend on $n, l$. For $l \rightarrow \infty$ with a fixed $n$, we have $\psi_{n l}^{\mathrm{CFT}} \rightarrow \sqrt{\frac{\Gamma\left(n+1+\Delta-\frac{d}{2}\right)}{\Gamma(n+1)}} l^{\Delta / 2-d / 4}$. For $n \rightarrow \infty$ with a fixed $l$, we have $\psi_{n l}^{\mathrm{CFT}} \rightarrow n^{\Delta-d / 2}$. For $n \rightarrow \infty$ with a fixed $l / n, \psi_{n l}^{\mathrm{CFT}} \rightarrow(n(n+l))^{\Delta / 2-d / 4}$. Note that these asymptotic behaviors are not exponential, but powers of $n, l$. Note also that the $(2 n+l+\Delta)^{a} \psi_{n l}^{\mathrm{CFT}} \rightarrow \infty$ for $n \rightarrow \infty$ if $a \geq 1$.

The other local operators in the $\mathrm{CFT}_{d}$ are given from $\mathcal{O}_{\Delta}(t, \Omega)$ by acting finite number of spacetime derivatives. If we are interested in scalar operators only, the derivatives should be composed by the time derivative and the Laplacian on $S^{d-1}$. The corresponding local states are given by

$$
\begin{aligned}
|\Omega, R\rangle_{\mathrm{CFT}}= & R\left(-i \frac{\partial}{\partial t},-\Delta_{S^{d-1}}\right) \\
& \times\left.\sum_{n, l, m} e^{i(2 n+l+\Delta) t} \psi_{n l}^{\mathrm{CFT}} Y_{l m}(\Omega) \hat{a}_{n l m}^{\dagger}|0\rangle\right|_{t=0} \\
= & \sum_{n, l, m} R(2 n+l+\Delta, l(l+d-2)) \\
& \times \psi_{n l}^{\mathrm{CFT}} Y_{l m}(\Omega) \hat{a}_{n l m}^{\dagger}|0\rangle
\end{aligned}
$$

where $R(x, y)$ is a polynomial of $x, y$. If we allow an infinite series for $R(x, y)$ it possibly represents a nonlocal state. We will take $R(x, y)$ as a polynomial of $x$ only and denote $R(x, y)$ as $R(x)$ for notational simplicity because the $y$ dependence is not relevant in the discussions below.

Because the energy of these states are divergent, it is important to consider the regularization of these states and the corresponding operators. Here, we consider the CFT and we can smear the local state over some region in time $t$ and space $\Omega$. For the finiteness of the energy we need to smear, at least, in time except for the free CFT case. ${ }^{15}$ Then, we can introduce, for example, the Gaussian factor $e^{-(2 n+l+\Delta)^{2} /\left(\omega_{c}\right)^{2}}$, where $\omega_{c} \gg 1$, by the time average over a period of $1 / \omega_{c}$, which gives the effective cutoff $\omega_{c}$ for $2 n+l+\Delta$ (and also the effective cutoff $l_{c}$ for $l$ by the averaging over $\Omega$ ).

With this regularization, we cannot regard the state (2.34) as a local state in general if the degree of the polynomial $R(x)$ is sufficiently large. This can be understood as follows. ${ }^{16}$ Let us take $R(x)=\sum_{q=0}^{q_{R}}(\text { iex })^{q} / q$ ! where $\epsilon$ is a real constant and the $q_{R}$ is the degree of the

\footnotetext{
${ }^{15}$ For the free scalar theory, the smearing over space only can give a finite energy state because there is no summation over $n$ for this case.

${ }^{16}$ As an example, let us consider the free field. The commutation relations at a fixed time, $\left[\frac{\partial^{q}}{\partial x^{q}} \phi(x), \pi\left(x^{\prime}\right)\right]=\frac{\partial^{q}}{\partial x^{q}} \delta\left(x-x^{\prime}\right)$, and $\left[R\left(-i \frac{\partial^{q}}{\partial x^{q}}\right) \phi(x), \pi\left(x^{\prime}\right)\right]=R\left(-i \frac{\partial^{q}}{\partial x^{q}}\right) \delta\left(x-x^{\prime}\right)$, where $R(x)=$ $\sum_{q=0}^{q_{R}}(\text { i } \in x)^{q} / q$ !, vanishe if $x \neq x^{\prime}$. For a smeared local field, the commutation relation is $\left[R\left(-i \frac{\partial^{q}}{\left.\partial x^{q}\right)} \int d y e^{-\left(\omega_{c}\right)^{2}(y-x)^{2}} \phi(y), \pi\left(x^{\prime}\right)\right]=\right.$ $R\left(-i \frac{\partial^{q}}{\partial x^{q}}\right) e^{-\left(\omega_{c}\right)^{2}\left(x-x^{\prime}\right)^{2}}$. By taking $\epsilon=x^{\prime}-x \gg 1 / \omega_{c}$, this is proportional to $e^{-\left(\omega_{c} \epsilon\right)^{2}}$ for $|\epsilon| \gg q_{R} / \omega_{c}$, then, exponentially suppressed. On the other hand, it is almost 1 for $|\epsilon| \ll q_{R} / \omega_{c}$.
} 
polynomial $R(x)$. Then, if $|i \in x|^{q_{R}+1} /\left(q_{R}+1\right) ! \ll 1$, this cannot be distinguished with $e^{i \epsilon x}$, which is the operator generating the translation, $t \rightarrow t+\epsilon$. Such an operation causes the nonlocality of $\mathcal{O}(\epsilon)$ for time, and then, the state (2.34) at $t=0$ is smeared over the ball-shape region with the size $\epsilon$ by the causality; thus it is not a local state. For a large $q_{R}$, this condition becomes $|\epsilon x| \ll q_{R}$ because $1 / q ! \sim e^{-q \log q}$. Because the maximum value of $2 n+l+$ $\Delta$ in (2.34) is $\mathcal{O}\left(\omega_{c}\right)$, the state (2.34) is supported in a space region of the size $|\epsilon|$ for this $R(x)$ if $|\epsilon| \ll q_{R} / \omega_{c}{ }^{17}$ This implies that, the state (2.34) is not necessary regarded as a local state, at least if $q_{R}$ is comparable to $\omega_{c}$.

Thus, if the cutoff $\omega_{c}$ is fixed or we consider the lowenergy effective theory below the energy $\omega_{c}$, the CFT local states (or operators) are effectively local only when $q_{R} \ll \omega_{c}{ }^{18}$ This is not inconsistent with the fact that local fields with arbitrary number of derivatives are local fields in a quantum field theory because $\omega_{c}$ can be taken as arbitrarily large. However, for the AdS/CFT, we will consider a subspace of the Hilbert space of CFT which introduces the largest $\omega_{c}$ because the bulk locality is meaningful only for low-energy states. (Low-energy effective theories for quantum field theories, in general, introduce the largest $\omega_{c}$.)

Denoting $\omega_{c}$ as the largest cutoff for the bulk picture, a CFT state (2.34) with the smearing with $\omega_{c}^{\prime}$ which satisfies $\omega_{c}^{\prime} \gg q_{R} \gg \omega_{c}$ is effectively a local state in the CFT. However, this state is constructed mostly from the very high-energy states with $n \gg \omega_{c}$. Such high-energy states can not be described in the local bulk picture.

We have seen that the (smeared) CFT local state at a point can effectively become the one at a different point by choosing $R(x)$ such that it mimics a translation. This might seem surprising; however, for field theories in the Minkowski spacetime, there is the Reeh-Schlieder theorem [44] which essentially states that the algebra of the local fields on a region in spacetime can generate any state approximately with an arbitrary precision (see, for example, [45]). Thus, the discussions above may be regarded as a (nonrigorous) explicit realization of the analog of the ReehSchlieder theorem.

Below, we will call the CFT states (2.34) (or operators) with $q_{R} \ll \omega_{c}$ [more precisely, $q_{R}=\mathcal{O}\left(\left(\omega_{c}\right)^{0}\right)$ ] as CFT effective local states (or operators) because we will only

\footnotetext{
${ }^{17}$ Instead of the translation, we can consider $R(x)$ which causes a smearing over the size $\epsilon$. For example, $R(x)=\sum_{q=0}^{q_{R} / 2}(\epsilon x)^{2 q} / q$ !, which is a truncation of $e^{-(\epsilon x)^{2}}$, is such an operation. For this $R(x)$ also, the state (2.34) is supported in a space region of the size $\epsilon$ if $\epsilon \ll q_{R} / \omega_{c}$.

${ }^{18}$ For the lattice field theory in a box of size $L=1$, with a lattice spacing $1 / \omega_{c}$, the $q_{R}$-derivative $\frac{\partial^{q_{R}}}{\partial t^{q}}$ is an operator which acts on fields on the $q_{R}$ sites and induces the nonlocality of the size $q_{R} / \omega_{c}$.
}

consider CFT operators and states with the regularization by the smearing over $1 / \omega_{c}$.

Note that the CFT effective local states only span a small subspace of the low-energy Hilbert space of the CFT. However, by the time evolution, a CFT effective local state at $t=t_{0} \neq 0$ gives a CFT state supported in a region at $t=0$, which is not given by effective local states on $t=0$. If we consider the CFT effective local states in the whole spacetime (or the spacetime region $\pi \leq t \leq \pi$ ) and consider the time evolution of them to the time slice $t=0$, then any low-energy state is expected to be given by them because in the low-energy theory the effective local states play the role of the local states.

\section{BULK LOCALITY IN AdS/CFT}

In this section, we will consider the relation between the bulk local states and the CFT (effective) local states. This topic is related to how the bulk locality emerges in the CFT. The bulk locality is seemingly paradoxical in the CFT point of view; however, we will see that there may be no paradox.

One of the "paradoxes" discussed in [29] is about the time-slice axiom which, essentially, says that there is no nontrivial operator which strictly commutes with any local field of CFT. However, from the bulk point of view, a boundary operator is obtained from the corresponding bulk field by taking it to the boundary with the appropriate scaling factor. This means that there is no bulk local field, strictly speaking. Indeed, the bulk local field is an approximate notion in the AdS/CFT correspondence because $N$ should be finite if the $\mathrm{CFT}_{d}$ is well defined as a $d$-dimensional field theory. Thus, this formal "paradox" is not a real problem. ${ }^{19}$ We will see below how bulk local fields appear in the CFT explicitly.

\section{A. Bulk local states from CFT states}

\section{Bulk states localized in radial direction}

Here, we will consider bulk states ${ }^{20}$ which are localized only in the radial direction at $\rho=\rho_{0}$ and extended in the angular direction, with $l=l_{0}, m=m_{0}$,

$$
\left|\rho_{0}, l=l_{0},, m=m_{0}\right\rangle \equiv \sum_{n} \psi_{n l_{0}}\left(\rho_{0}\right) \hat{a}_{n l_{0} m_{0}}^{\dagger}|0\rangle,
$$

with the regularization discussed above. These states can be obtained from the bulk local states by an appropriate

\footnotetext{
${ }^{19}$ Indeed, as we will see below, the boundary operators are the CFT effective local operators, which are in a subset of the CFT local operators. Thus, the bulk operator can commute with the boundary operators effectively.

${ }^{20}$ The bulk states and the CFT states are identical, of course. (In the low energy, it is identical and the CFT may be the definition of the theory above the cutoff scale.) Here the "bulk state" means that we consider a state, from the bulk point of view, which is obtained by acting a bulk local field on the vacuum.
} 
averaging over angular directions $\Omega$. Note that for $\rho_{0}=0$ this is the local state at $\rho=0$ (which implies $l_{0}=0$ ) and for $\rho_{0} \neq 0$ this is localized in the radial direction, but extended in the angular directions. For the latter case, if we take $l_{0}=0$ in particular, the states are uniform in $S^{d-1}$.

Let us consider the time evolution of this state. At time $t$, this state becomes

$$
\begin{aligned}
& e^{i \hat{H} t}\left|\rho_{0}, l=l_{0}, m=m_{0}\right\rangle \\
& \quad=\sum_{n} e^{i\left(2 n+l_{0}+\Delta\right) t} \psi_{n l_{0}}\left(\rho_{0}\right) \hat{a}_{n l_{0} m_{0}}^{\dagger}|0\rangle .
\end{aligned}
$$

Because $\psi_{n l}$ is not singular for finite $n$, the dominant contributions in the summation are from $n \gg 1$, and, by using (2.25), the phase of each of the contributions becomes

$$
\begin{aligned}
& e^{i\left(2 n+l_{0}+\Delta\right) t} \cos \left(\left(2 n+l_{0}+\Delta\right) z_{0}-\frac{\pi}{2}\left(\Delta-\frac{d}{2}+\frac{1}{2}\right)\right) \\
& =\frac{1}{2} e^{i\left(2 n+l_{0}+\Delta\right)\left(t+z_{0}\right)-\frac{\pi}{2}\left(\Delta-\frac{d}{2}+\frac{1}{2}\right)}+\frac{1}{2} e^{i\left(2 n+l_{0}+\Delta\right)\left(t-z_{0}\right)+\frac{\pi}{2}\left(\Delta-\frac{d}{2}+\frac{1}{2}\right)},
\end{aligned}
$$

where $z_{0}=\pi / 2-\rho_{0}$. Thus, at time $t$, the states becomes localized at $z=z_{0} \pm t$. This is consistent with the fact that the lightlike trajectory in the radial direction of the $A d S$ spacetime is $z=z_{0} \pm t$ because the localized state in the radial direction has an infinite energy (without regularization). ${ }^{21}$ One might think that this is inconsistent with the HKLL reconstruction of the bulk local state at the center for the global AdS [16] because the smearing function used in [16] is supported in the region $-\pi / 2 \leq t \leq \pi / 2$. However, because the smearing function is $K(t)=(\cos t)^{\Delta-d}$ or $K=(\cos t)^{\Delta-d} t, \int d t K(t)$ is divergent near $t= \pm \pi / 2$ for $\Delta \leq d-1$. Thus, the integration is indeed localized at these points. ${ }^{22}$

Let us concentrate on the $l_{0}=0$ case and consider what the corresponding state is as a linear combination of the CFT local states (2.34) with regularization. Because of the rotational symmetry, the corresponding state should be (2.34) with $l=0$

\footnotetext{
${ }^{21}$ For $\Delta=d / 2$ which saturates the Breitenlohner-Freedman bound [46] in the bulk picture, the particle in the bulk travels through the lightlike trajectory. Indeed, for $\Delta=d / 2=1$, we can check that $\psi_{n 0}(\rho=0)=C e^{i(2 n+\Delta) \pi / 2} \psi_{n 0}^{\mathrm{CFT}}$, where $C$ is an $n$-independent constant. This means that the bulk local state at $\rho=0, t=0$ is obtained by the CFT local state averaged over $S^{1}$ at $t=-\pi / 2(\bmod \pi)$. For $\Delta=d / 2$ and $d>2$, there will appear some kinematic factor.

${ }^{22}$ The divergence is regularized by the smearing of the bulk local operator. For $\Delta>d-1$, some modifications of the discussion in [16] would be needed.
}

$$
|l=0, R\rangle_{\mathrm{CFT}} \equiv \sum_{n} R(2 n+\Delta) \psi_{n 0}^{\mathrm{CFT}} \hat{a}_{n 00}^{\dagger}|0\rangle
$$

with an appropriate $R(x)$. Thus, the $l=0$ state is uniform in the space $S^{d-1}$ and cannot be described by the CFT state supported in any subregion of the space $S^{d-1}$. This implies that the bulk local state at the center of AdS space is supported in whole space $S^{d-1}$ and then the version of the subregion duality may not be correct.

For general $l$, we will see how the following CFT state constructed from the CFT states (2.34) integrating over the angular direction,

$$
\begin{aligned}
\left|l=l_{0}, m=m_{0}, R\right\rangle_{\mathrm{CFT}} & \equiv \int d \Omega Y_{l_{0}, m_{0}}^{*}(\Omega)|\Omega, R\rangle_{\mathrm{CFT}} \\
& =\sum_{n} R\left(2 n+l_{0}+\Delta\right) \psi_{n l_{0}}^{\mathrm{CFT}} \hat{a}_{n l_{0} m_{0}}^{\dagger}|0\rangle,
\end{aligned}
$$

realizes the bulk state localized in the radial direction (3.1) by choosing $R(x)$ appropriately. We will also see how the bulk locality in the radial direction is realized in the CFT for this example.

In order to have a bulk state which is localized in the radial direction (3.1) and its $\rho$-derivative, we need to choose the polynomial $R$ in (3.5) such that the phase factor in the large $n$ is reproduced, i.e.,

$$
\begin{aligned}
& R\left(2 n+l_{0}+\Delta\right) \psi_{n l_{0}}^{\mathrm{CFT}} \\
& \quad \sim \exp \left( \pm i\left(\left(2 n+l_{0}+\Delta\right) z_{0}-\frac{\pi}{2}\left(\Delta-\frac{d}{2}+\frac{1}{2}\right)\right)\right)
\end{aligned}
$$

for $1 \ll n \leq \omega_{c}$, where the right-hand side is the asymptotic behavior of $\psi_{n l_{0}}\left(\rho_{0}\right)$. This is possible because $R$ is an arbitrary polynomial and the range of $n$ considered here is finite. Indeed, if we take $R(x)=\sum_{q=0}^{q_{R}}\left(i z_{0} x\right)^{q} / q$ ! where $z_{0} \ll q_{R} / \omega_{c}$, which is effectively a time translation operator, the state (3.5) reproduces the phase (3.6) and then the state is localized at $\rho=1 / z_{0}$. We note that $q_{R}$ is, at least, comparable with $\omega_{c}$ which is very large, thus the CFT state which corresponds to the bulk local state contains a large number of derivatives. Note that with this choice of $R(x)$, the CFT local state $|\Omega, R\rangle_{\mathrm{CFT}}$ used in (3.5) is not a CFT effective local state.

Thus, the CFT state (3.5) with the appropriate choice of $R(x)$ describes the bulk local states (3.1). This is possible because of the large degrees of freedom of the large $N$ gauge theory. The different states at different $\rho$, which are orthogonal to each other, are embedded in the $\mathcal{O}\left(N^{2}\right)$ internal degrees of freedom of the CFT, which essentially correspond to the label $n$ and the $n$-dependence of the state is changed by the time derivatives. A linear combination of the CFT local states with the large number of time 
derivatives corresponds to the bulk state away from the boundary.

\section{Examples of bulk local states from CFT states}

We have seen that the bulk local state at the center corresponds to the CFT state which is supported on whole space $S^{d-1}$ uniformly. This implies that a generic bulk local state also corresponds to a CFT state which is supported on whole space, because of continuity.

On the other hand, some particular bulk local states can correspond to CFT states which are supported on a subregion in the space as we will see below. ${ }^{23}$ Let us consider the CFT state (2.34) at $\Omega=\Omega_{0}$ with the regularization,

$$
\begin{aligned}
\mid \Omega & \left.=\Omega_{0}, R\right\rangle_{\mathrm{CFT}} \\
& =\sum_{n, l, m}^{2 n+l+\Delta<\omega_{c}, l<l_{c}} R(2 n+l+\Delta) \psi_{n l}^{\mathrm{CFT}} Y_{l m}\left(\Omega_{0}\right) \hat{a}_{n l m}^{\dagger}|0\rangle .
\end{aligned}
$$

This state is a CFT local state smeared over the time with the length scale $1 / \omega_{c}$ and over the space with the length scale $1 / l_{c}$. Here, we take the parameters of the regularizations as $1 \ll l_{c} \ll \omega_{c} .\left(1 / \omega_{c}\right.$ can be the smallest one, which may be related to the Planck length and $1 / l_{c}$ will be an arbitrary (very small) length scale, but much larger than $1 / \omega_{c}$.)

If this CFT state corresponds to the bulk local state (2.22) at $\rho=\rho_{0}, \Omega=\Omega_{0}$, the phase factor should be reproduced. For this, we need to take

$$
R(x)=\sum_{q=0}^{q_{c}}\left(i z_{0} x\right)^{q} / q !,
$$

where $z_{0} \ll q_{c} / \omega_{c}$ as in (3.6). Then, if we neglect the $1 / l_{c}$ suppressed terms, the overlap between the (normalized) CFT state (3.7) and the bulk local state (2.22) at $\rho=\rho_{1}$, $\Omega=\Omega_{1}$, with the regularization by $\omega_{c}$,

$$
\begin{aligned}
& \left\langle\rho_{1}, \Omega_{1} \mid \Omega=\Omega_{0}, R\right\rangle_{\mathrm{CFT}} \\
& \quad=\sum_{n, l, m}^{2 n+l+\Delta<\omega_{c}, l<l_{c}} R(2 n+l+\Delta) \psi_{n l}^{\mathrm{CFT}} \psi_{n l}\left(\rho_{0}\right) \\
& \quad \times Y_{l m}^{*}\left(\Omega_{1}\right) Y_{l m}\left(\Omega_{0}\right),
\end{aligned}
$$

divided by the normalization factors of the two states vanishes ${ }^{24}$ except at $\rho_{0}=\rho_{1}$ up to $1 / \omega_{c}$ and at $\Omega_{0}=\Omega_{1}$ up to $1 / l_{c}$ because $\sum_{l, m} Y_{l m}^{*}\left(\Omega_{0}\right) Y_{l m}\left(\Omega_{1}\right) \sim \delta\left(\Omega_{0}-\Omega_{1}\right)$.

\footnotetext{
${ }^{23}$ The bulk local state constructed here is a local state with the resolution $1 / l_{c}$, but not a local state with the resolution $1 / \omega_{c}$. Thus, precisely speaking, the bulk local state constructed here is not an effectively local state.

${ }^{24}$ For $\omega_{c} \sim l_{c}$, the asymptotic behavior of $\psi_{n l}$ is complicated [47], thus we can not construct a generic bulk local state from a CFT local state.
}

Thus, this CFT state (with the finite number of the spacetime derivatives) corresponds to a bulk local state at $\rho=\rho_{0}=1 / z_{0}$ and $\Omega=\Omega_{0}$ smeared over the small spacetime region.

Note that the condition $1 \ll l_{c} \ll \omega_{c}$ implies that the corresponding bulk local state may have momentum only along the radial direction. ${ }^{25}$ This interpretation is consistent with the fact that the CFT state is the bulk local state at $z=z_{0}$ which is moving into the center along the radial direction from the boundary at $t=-z_{0}$. The bulk local state at the center with $l=0$ can be obtained by the superposition of such CFT states with $z_{0}=\pi / 2$ averaging over the whole space.

Note also that this CFT state, which is approximated as

$$
\begin{aligned}
\left|\Omega=\Omega_{0}, R\right\rangle_{\mathrm{CFT}} \approx & \sum_{n, l, m}^{2 n+l+\Delta<\omega_{c}, l<l_{c}} e^{i z_{0}(2 n+l+\Delta)} \psi_{n l}^{\mathrm{CFT}} \\
& \times Y_{l m}\left(\Omega_{0}\right) \hat{a}_{n l m}^{\dagger}|0\rangle,
\end{aligned}
$$

is not a CFT effective local state because of the large number of derivatives in $R$ which are regarded as a time translation operator by $z_{0}$. Thus, this CFT state is supported on the approximately sphere-shape $\left(S^{d-2}\right)$ region with radius $z_{0}$ in the space $S^{d-1}$, as we will explicitly see below. The overlap between this state and a CFT local state at $\Omega=\Omega_{1}$ associated with a polynomial $\tilde{R}(x)$ is

$$
\begin{aligned}
\mathrm{CFT} & \left\langle\Omega=\Omega_{1}, \tilde{R} \mid \Omega=\Omega_{0}, R\right\rangle_{\mathrm{CFT}} \\
\approx & \sum_{n, l, m}^{2 n+l+\Delta<\omega_{c}, l<l_{c}} e^{i z_{0}(2 n+l+\Delta)} \tilde{R}^{*}(2 n+l+\Delta)\left(\psi_{n l}^{\mathrm{CFT}}\right)^{2} \\
& \times Y_{l m}^{*}\left(\Omega_{1}\right) Y_{l m}\left(\Omega_{0}\right) \\
= & \sum_{l, m}^{l<l_{c}}\left(\sum_{n}^{2 n+l+\Delta<\omega_{c}} e^{i z_{0}(2 n+\Delta)} \tilde{R}^{*}(2 n+l+\Delta)\left(\psi_{n l}^{\mathrm{CFT}}\right)^{2}\right) \\
& \times Y_{l m}^{*}\left(\Omega_{1}\right) e^{i z_{0} l} Y_{l m}\left(\Omega_{0}\right) .
\end{aligned}
$$

We take $\left|\Omega=\Omega_{1}, \tilde{R}\right\rangle_{\mathrm{CFT}}$ as a CFT effective local state at $\Omega=\Omega_{1}$, i.e., the degree of the polynomial $\tilde{R}(x)$ is much less than $\omega_{c}$. Then, the summation of $n$ in the last line of (3.11) is almost canceled by the phase factor and is exponentially suppressed, except for the terms for $n<\mathcal{O}\left(1 / z_{0}\right) .{ }^{26}$ These remaining terms are not exponentially large for large $l$ because $\left(\psi_{n l}^{\mathrm{CFT}}\right)^{2} \sim l^{\Delta-d / 2}$. We also see that $\sum_{l, m}^{l<l_{c}} Y_{l m}^{*}\left(\Omega_{1}\right) e^{i z_{0} l} Y_{l m}\left(\Omega_{0}\right)$ almost vanish except

\footnotetext{
${ }^{25} 1 \ll l_{c} \ll \omega_{c}$ implies $1 \ll l_{c} \ll n_{c}$ where $n_{c}$ is the largest $n$ in the sum. Note also that the momentum is incoming in the radial direction but by changing the $R(x)$ to $R(-x)$, it becomes outward.

${ }^{26}$ The overlap (3.11) is small, if we normalize $\left|\Omega=\Omega_{1}, \tilde{R}\right\rangle_{\mathrm{CFT}}$ by choosing the overall factor of $\tilde{R}(x)$ appropriately, because of the cancellation. However, the number of the independent CFT effective local states are large due to the choice of $\tilde{R}(x)$.
} 
$\Omega_{1} \in S_{z_{0}}^{d-2}\left(\Omega_{0}\right)$ up to $1 / l_{c}$ corrections where $S_{z_{0}}^{d-2}\left(\Omega_{0}\right)$ is the sphere-shape ( $S^{d-2}$ ) region whose center is at $\Omega=\Omega_{0}$ with radius $z_{0}$ in the space $S^{d-1}$. To see this, let us consider the free CFT, i.e., $\Delta=d / 2-1$, although it is not a holographic CFT. For this case there is no summation over $n$ and the overlap (3.11) becomes

$$
\begin{aligned}
\mathrm{CFT}\left\langle\Omega=\Omega_{1}, \tilde{R} \mid \Omega=\Omega_{0}, R\right\rangle_{\mathrm{CFT}} \\
=\sum_{l, m}^{l<l_{c}}\left(e^{i z_{0} \Delta} \tilde{R}^{*}(l+d / 2-1) \frac{2 d-1}{\pi(l+d / 2-1)}\right) \\
\quad \times Y_{l m}^{*}\left(\Omega_{1}\right) e^{i z_{0} l} Y_{l m}\left(\Omega_{0}\right),
\end{aligned}
$$

which should almost vanish except the case that $\Omega_{0}$ can be reached at $t=z_{0}$ by a light ray, which is described by the free CFT, emanating from $\Omega=\Omega_{1}$ at $t=0$. This means that $\sum_{l, m}^{l<l_{c}} Y_{l m}^{*}\left(\Omega_{1}\right) e^{i z_{0} l} Y_{l m}\left(\Omega_{0}\right)$ has the property stated above. Therefore, the CFT state (3.10) is supported on the sphere region from the CFT point of view although it is the bulk local state from the bulk point of view. ${ }^{27}$

In order to understand more clearly how the bulk local state appears from the CFT let us consider $d=2$ case. For this case, the CFT state at $t=0$ is supported on two points $S^{0}$ which is a time(-reversed) evolution of the CFT effective local state at $\Omega=\Omega_{1}, t=z_{0}$. Then, one might think that the particles at the two points are entangled and regarded as an Einstein-Podolsky-Rosen (EPR) pair. However, These two particles are just a superposition although the EPR pair is a two-particle state. Thus, the bulk local state is a nonlocal state in the CFT and this nonlocality is related to the basis change of the states, which dose not keep the locality. This also means that the state is an entangled state.

\section{B. CFT states supported in a space region from bulk local states}

As we have seen, the bulk local state is, in general, reconstructed from the states in the CFT supported in the whole space $S^{d-1}$. (This fact was also known in the HKLL reconstruction in the global coordinate [16].) However, the CFT effective local states should be supported in some region in the bulk, in the bulk point of view, because of the causality. For example, the CFT local operator at the north pole of $S^{d-1}$ and the one at the south pole should commute with each other (if the difference of time is shorter than $\pi$ ) by the causality in the CFT. ${ }^{28}$ This is impossible if the corresponding bulk states are extending in the bulk sufficiently because propagation in the bulk can connect two

\footnotetext{
${ }^{27}$ For $z_{0}=\pi$, the CFT state is a CFT effectively local state at the opposite point $\left(\bar{\Omega}_{0}\right)$ to $\Omega=\Omega_{0}$ in $S^{d-1}$ as we can see it explicitly by using $e^{i \pi l} Y_{l m}\left(\Omega_{0}\right)=Y_{l m}\left(\bar{\Omega}_{0}\right)$.

${ }^{28}$ Our smearing of the fields is not restricted on a region, but it is like a Gaussian. Thus, the commutator vanishes with some terms suppressed by the regularization parameters.
}

boundary regions faster than what the CFT requires, as shown in Appendix. Note that there is only one free scalar field in the bulk corresponding to the large $N$ CFT fields, i.e., the degrees of freedom of the bulk theory is one [or $\mathcal{O}(1)]$, not $\mathcal{O}\left(N^{2}\right)$. Thus, if the propagation in the bulk connects two spacetime points, two generic local operators at these points will have a nonzero commutator.

In this section, we will study bulk interpretations of the CFT effective local state and the CFT states which are supported in a region and see how these states extend in the bulk. $^{29}$

\section{CFT effectively local states}

Here, we will explicitly consider bulk interpretation of the CFT local state $\left|\Omega_{0}, R\right\rangle_{\mathrm{CFT}}$ at $\Omega=\Omega_{0}$ with the regularization by the smearing for the time direction and the space $\left(S^{d-1}\right)$ direction with the resolutions given by $1 / \omega_{c}$ and $1 / l_{c}$, respectively

$$
\begin{aligned}
\left|\Omega_{0}, R\right\rangle_{\mathrm{CFT}}= & \sum_{n, l, m}^{2 n+l+\Delta<\omega_{c}, l<l_{c}} R(2 n+l+\Delta) \\
& \times \psi_{n l}^{\mathrm{CFT}} Y_{l m}\left(\Omega_{0}\right) \hat{a}_{n l m}^{\dagger}|0\rangle .
\end{aligned}
$$

This state is a CFT effective local state by requiring that the degree $q_{R}$ of the polynomial $R(x)$ is small $\left(\omega_{c} \gg q_{R}\right)$ and the derivatives do not change the large $n$ behavior of the phase factor. Here, we concentrate on the case that the smearing for the space direction is much larger than the one for the time direction, which means $1 \ll l_{c} \ll \omega_{c}{ }^{30}$ The overlap between this state and the bulk local state (2.22) at $\rho=\rho_{1}, \Omega=\Omega_{1}$ is given by (3.9) and, for this case, the phase factor in the sum for the $n$ is

$$
\begin{aligned}
& R(2 n+l+\Delta) \psi_{n l}^{\mathrm{CFT}} \psi_{n l}\left(\rho_{1}\right) \\
& \quad \sim \cos \left((2 n+l+\Delta) z_{1}-\frac{\pi}{2}\left(\Delta-\frac{d}{2}+\frac{1}{2}\right)\right),
\end{aligned}
$$

for $l \ll n \leq \omega_{c}$. Thus, the overlap almost vanishes except $z_{1}\left(=\pi / 2-\rho_{1}\right)=0$ up to $1 / \omega_{c}$ and $\Omega_{0}=\Omega_{1}$ up to $1 / l_{c}$. This means that the CFT effective local states corresponds to the bulk local states near the point on the boundary. In other words, the CFT primary states (or fields) with a sufficiently small number of derivatives (and a low conformal dimension) live on the boundary of the bulk theory. This statement is a refinement of the statement "CFT lives

\footnotetext{
${ }^{29}$ In [48], similar discussions have been done for the Euclidian time evolution.

${ }^{30}$ We can also consider the case with $l_{c} \sim \omega_{c}$ or the case with the smearing for the time direction only by using the results in [47]. Here, we consider the case with $1 \ll l_{c} \ll \omega_{c}$ only because it is simpler and sufficient for showing where in the bulk the CFT state extends.
} 
on the boundary of AdS" in AdS/CFT correspondence although the CFT corresponds to the whole bulk theory.

If we change the high energy cutoff $\omega_{c}$ lower, the CFT effective local state when this cutoff extends in the radial direction in the bulk for $z<\mathcal{O}\left(1 / \omega_{c}\right)$. This may be a kind of a realization of the $\mathrm{UV} / \mathrm{IR}$ relation.

\section{CFT states supported in a region}

Let us consider CFT states effectively supported in a space $\left(S^{d-1}\right)$ region $A$, and we call the subspace of the Hilbert space spanned by them as $\mathcal{H}_{A}^{\text {eff }}$. Because we consider only the lowenergy states, the states are really supported in the spacetime region which is given by extending the space region to the time direction with the width $1 / \omega_{c}$. Note that the CFT (formal) local states, which are obtained by the CFT local operators acting on the vacuum, are not included in $\mathcal{H}_{A}^{\text {eff }}$ generically because they are effectively nonlocal even if they are smeared over the region $A$. Note also that the linear combinations of the CFT effective local states in the space region $A$ are in $\mathcal{H}_{A}^{\text {eff }}$, however, there exist other states in $\mathcal{H}_{A}^{\text {eff }}$, corresponding to the states inside the bulk, as we have seen. Indeed, the CFT state (3.10) is not a CFT effective local state, but is supported in the $S^{d-2}$ sphere with radius $z_{0}$ around $\Omega=\Omega_{0}$. Thus, this state is in $\mathcal{H}_{A}^{\text {eff }}$ if we choose $z_{0}$ appropriately small and $\Omega_{0} \in A$.

Note that the CFT states which are supported in the $S^{d-2}$ sphere around $\Omega=\Omega_{0}$ with radius $z_{0}$, or the ball including the inside of this sphere, can not correspond to a bulk state, including a bulk local state at $z>z_{0}, \Omega=\Omega_{0}$, because of the causality discussed above and the results in Appendix. The CFT state (3.10) which corresponds to a bulk local state at $z=z_{0}, \Omega=\Omega_{0}$, has the maximum value for $z$ under this causality bound for the states in $\mathcal{H}_{A}^{\text {eff }}$.

Let us consider the bulk states correspond to the CFT states supported in this ball shaped region $A$ (at $t=0$ ). These CFT states will be generated by considering the all CFT effective local states in the causal diamond of the space region $A$ (the boundary domain of dependence of $A$ ) and the corresponding CFT states at $t=0$ by the time evolution. ${ }^{31}$

We expect that the CFT effective local states at a point $p$ in the causal diamond can represent any bulk local state, which can move in various directions, at the point $p$ which are on the boundary of the bulk spacetime. Thus, the region $a$ in the $t=0$ slice in the bulk (which can be reached by the bulk local states in the causal diamond by the time evolution) is the bulk region corresponding to the region $A$ and we can see that this region $a$ coincides with the causal wedge of $A$. This means that the CFT states supported in the region $A$ are given by the bulk states supported in the causal wedge of $A$.

\footnotetext{
${ }^{31} \mathrm{CFT}$ states which enter in the causal diamond by the time evolution are also supported in the region $A$, however, the corresponding CFT states at $t=0$ are represented by the CFT states in the causal diamond also.
}

This is similar to the version of the subregion duality, however, there is no inverse of this, thus this is not like a duality. As we have seen, there are some bulk states supported in the causal wedge of $A$ which can not be given by the CFT states supported in the region $A$. Indeed, the CFT state (3.10) corresponds to a bulk local state at $z=z_{0}, \Omega=$ $\Omega_{0}$ moving along the radial direction and it may not be possible to correspond to a bulk local state at the point moving in a different direction. ${ }^{32}$ One might think that by changing the phases in (3.10) one could build a wave packet that describes an excitation moving in any direction and is able to represent any state in the causal wedge of A. But, if the phases are changed by hand (or further smearing over the radial direction), then the state is no longer supported in CFT region A, generically. Thus, it is impossible to construct such wave packet from the CFT states in A.

Thus, a bulk local state is decomposed to the bulk local states moving in particular directions, each of which are represented by the CFT state (3.10) or the rotation of it. Some of these rotated CFT states (3.10) are supported in region $A$ and the others are not supported in $A$. The bulk local state constructed from the CFT states supported in a region $A$ is a sum of such CFT states, but only for the ones supported in the region $A$. This also implies that bulk local states (or operators) at a same bulk point constructed from CFT states supported in different regions are different even in the low energy (gravity) theory. ${ }^{33}$ Note that in the quantum error correction code proposal [29], two such bulk local states were assumed to be same in the code subspace, which may correspond to the subspace of the low-energy gravity theory. Thus, in our example, the quantum error correction code proposal may not be realized.

\section{Entanglement wedge or causal wedge?}

As we have seen, for the connected region $A$, the CFT states supported in the region $A$ are given by the bulk states supported in the causal wedge of $A$. The causal wedge of $A$ and entanglement wedge of $A$ are same for this case. On the other hand, these two wedges can be different for a region $A$ which is a sum of disconnected regions $A_{1}$ and $A_{2}$, i.e., $A=$ $A_{1} \cup A_{2}$ and $A_{1} \cap A_{2}=\varnothing$. For this case, there are bulk states which are not contained in bulk states supported in $a_{1} \cup a_{2}$ where $a_{i}$ is the causal wedge of $A_{i}$. Let us consider,

\footnotetext{
${ }^{32}$ One might think that the superposition of different local states can move another direction. However, such superposition is not a bound state and can move in different directions separately. In particular, by averaging the CFT states over a time period of $1 / l_{c}$ near $t=z_{0}$. we obtain a CFT state corresponding to bulk local states moving in all directions. The bulk local state at $z=z_{0}, t=0$, which is a small part of it, is moving in the radial direction.

${ }^{33}$ The bulk local state moving in some direction is not a local state with the resolution $1 / \omega_{c}$. Thus, precisely speaking, the bulk local state can not be constructed from the CFT states supported in the subregion.
} 
for example, the case where $A_{i}$ are very small regions and are regarded as points. Then, there is a spacetime point $p$ of the CFT (or the boundary of the bulk) which is a midpoint of $A_{1}$ and $A_{2}$ in space and at the (past) time such that the point $p$ is connected to $A_{1}$ and $A_{2}$ by lightlike trajectories. Then the time evolution to $t=0$ of a CFTeffective local state at $p$ may be supported in $A=A_{1} \cup A_{2}$ from the CFT point of view. In the bulk viewpoint, a bulk state at $p$ can move toward the center (i.e., in the radial direction) or toward $A_{i}$ in space. Moreover, it can move in any direction between these and will reach different bulk points at $t=0$. Then, for the $d=2$ case, the bulk state at $t=0$ will be supported on a minimal surface (curve) in the bulk connecting $A_{1}$ and $A_{2}$ because the minimal surface is the boundary of the causal wedge of the segment which is given by the geodesics between $A_{1}$ and $A_{2}$. For general $d$, this statement will be correct although the minimal surface is degenerated. Thus, for this $A$, the CFT states supported in the region $A$ correspond to the bulk states supported in the sum of the causal wedge of $A$ and this region given by the minimal surface, which is not the entanglement wedge. $^{34}$

Now, let us consider the case where $A_{i}$ are not small regions. For this case, the bulk states at $t=0$ will be supported in the region which is the sum of the (degenerate) minimal surface between the all possible points in $A_{1}$ and $A_{2}$. This region is expected to be the bulk region whose boundaries are $A_{i}$ and the minimal surface connecting these in the space at $t=0$. Note that the entanglement wedge is the region surrounded by the Ryu-Takayanagi surface [50] for $A$, which is this region or the causal wedge of $A$, depending on the area of the minimal surfaces. We will call the sum of regions surrounded by any possible minimal surface whose boundaries are in the boundary of $A$ as the minimal surface wedge of $A$. Then, the minimal surface wedge may be the bulk region corresponding to $A$, i.e., any CFT state supported in $A$ may be expressed by a bulk state supported in the minimal surface wedge of $A .^{35}$

Note that it is known that the causal wedge is always inside the entanglement wedge for our case. However, the bulk local state at a space point $p$ in the causal wedge constructed from $A_{1}$ (or $A_{2}$ ) only is different from the above bulk local state at the same point $p$ constructed from both $A_{1}$ and $A_{2}$. According to [50], the entanglement entropy is proportional to the area of the Ryu-Takayanagi surface. It is interesting to understand this Ryu-Takayanagi formula explicitly in the view point of this paper.

\section{Comments on subregion duality and quantum error correction}

As we have seen, the version of the subregion duality is not valid in our analysis. A bulk local state corresponds to a CFT

\footnotetext{
${ }^{34}$ In [49], some related ideas were explored from a different perspective.

${ }^{35} \mathrm{We}$ expect that this is true for any region $A$.
}

state supported in whole space, although a CFT state supported in a region $A$ will correspond to a bulk state supported in the minimal surface wedge of $A$. This is possible because a bulk state constructed from CFT states in a region $A$ is different from a bulk state at the same point constructed from CFT states in a region $A^{\prime}$ which is different from $A$.

Our analysis is essentially the same as the global AdS reconstruction of HKLL. Instead of it, the AdSRindler reconstruction was also discussed in [16]. This reconstruction was used to argue that the quantum error correction is relevant for quantum gravity in [29]. However, the AdS-Rindler wedge only covers a part of the AdS spacetime and we need continuum spectrum for the mode expansion for a construction of the smearing function for it. The spectrum of the CFT is discrete, where the Hamiltonian is the dilatation. ${ }^{36}$ Thus, we need infinite energy modes of the CFT for the AdS-Rindler reconstruction ${ }^{37}$; the bulk state from the AdS-Rindler reconstruction is unclear in the CFT states, in particular for the low-energy theory.

If we forget about the CFT states and the Hamiltonian, based on which we have discussed low-energy theory, the AdS-Rindler Hamiltonian will not be a problem and there will be duality between the subregions of the bulk and CFT, like the AdS-Poincaré reconstruction. However, if we consider the two different regions, $A$ and $A^{\prime}$, the AdS-Rindler reconstructions depend on the different Hamiltonians on the two regions and it is unclear how to compare the two bulk local states constructed in the different regions in the low-energy theories; in particular the two bulk local states will not be equivalent in the low-energy theory. Thus, it is not justified to use the AdS-Rindler reconstruction for the discussion on the bulk local operators for such cases.

In our analysis, a CFT state supported in a ball-shaped region $A$ will correspond to a bulk state supported in the causal wedge of $A$, which also appears in the AdS-Rindler reconstruction; however, two bulk local states at the same spacetime point constructed in different regions are different even in the low-energy theory. Thus, the theory does not have the structure of quantum error correction codes discussed in [29].

The bulk local operator of the AdS-Rindler reconstruction will reproduce the bulk correlation function. However, the correlation function with the same bulk local operator insertions will be different from each other for the AdS-Rindler reconstruction and the global reconstruction. This is because the AdS-Rindler reconstruction used the eigenmodes with the (in-going) boundary condition in the bulk, then it reproduces the bulk correlation function with

\footnotetext{
${ }^{36}$ In order to define the bulk operator, we need to choose the Hamiltonian because the bulk local operator will be defined in the low-energy theory and the low-energy states on which the bulk local operators act are defined by the Hamiltonian.

${ }^{37}$ The continuum spectrum is due to the boundary condition for the Rindler wedge although the CFT states satisfies the periodic boundary condition for $S^{d-1}$.
} 
the boundary condition, which is absent for the global reconstruction. Thus, the bulk local operator of the AdSRindler reconstruction should be different from the bulk local operator of the global reconstruction even in the low energy.

\section{ACKNOWLEDGMENTS}

S. T. would like to thank Kanato Goto, Lento Nagano, Yu Nakayama, Yausnori Nomura, Sotaro Sugishita, Yuki Suzuki, Tadashi Takayanagi, Kotaro Tamaoka, and Koji Umemoto for useful discussions. S. T. would like to thank the Yukawa Institute for Theoretical Physics at Kyoto University. Discussions during the workshop YITP-T-1903 "Quantum Information and String Theory 2019" were useful to complete this work. This work was supported by JSPS KAKENHI Grant No. 17K05414.

\section{APPENDIX: ON THE CAUSALITY ON AdS/CFT}

The metric of $\operatorname{AdS}_{d-1}$ spacetime is given by

$$
d s_{\mathrm{AdS}}^{2}=\frac{1}{\sin ^{2}(z)}\left(-d t^{2}+d z^{2}+\cos ^{2}(z) d \Omega_{d-1}^{2}\right) .
$$

where $0 \leq z \leq \pi / 2$.
Let us consider a lightlike trajectory along the radial direction from a bulk point near the north pole $(z=\epsilon$, $\theta=0)$ at $t=0$ to the south pole $(z=0, \theta=\pi)$ where $\theta$ is an angle variable of $S^{d-1}$ satisfying $0 \leq \theta \leq \pi$. For this trajectory, $d t=d z$, and it will be at the south pole at $t=\pi-\epsilon$. Instead of this, let us consider a lightlike trajectory along the angular direction from a bulk point on the boundary near the north pole $(z=0, \theta=\epsilon)$ at $t=0$ to the south pole $(z=0, \theta=\pi)$ with $d z=0$ satisfying $d t=d \theta$, thus it will also be at the south pole at $t=\pi-\epsilon$. Note that this lightlike trajectory is regarded as the one in the $\mathrm{CFT}_{d}$ on $S^{d-1}$ as a space.

Thus, the CFT state supported in a ball-shaped region of size $\epsilon$ centered at the north pole of $S^{d-1}$ cannot correspond to the bulk local state at $z>\epsilon, \theta=0$ because of the causality of the CFT.

The following is not relevant for the discussions in this paper, but, we can consider a lightlike trajectory from a bulk point near the north pole $(z=\epsilon, \theta=0)$ at $t=0$ to a point near the south pole $\left(z=\epsilon^{\prime}, \theta=\pi\right)$ not along the radial direction. It satisfies $d t=d \theta \sqrt{\frac{d z^{2}}{d \theta^{2}}+\cos ^{2}(z(\theta))}>$ $d \theta \cos (z(\theta))$. Thus it will be at the point after $t=\pi-\pi\left(\epsilon^{\prime}\right)^{2} / 2$, for $\epsilon \geq \epsilon^{\prime}$, where we assumed $\epsilon \ll 1$.
[1] J. M. Maldacena, The large N limit of superconformal field theories and supergravity, Int. J. Theor. Phys. 38, 1113 (1999) [Adv. Theor. Math. Phys. 2, 231 (1998)].

[2] S. B. Giddings, Holography and unitarity, J. High Energy Phys. 11 (2020) 056.

[3] S. Banerjee, J. W. Bryan, K. Papadodimas, and S. Raju, A toy model of black hole complementarity, J. High Energy Phys. 05 (2016) 004.

[4] S. S. Gubser, I. R. Klebanov, and A. M. Polyakov, Gauge theory correlators from noncritical string theory, Phys. Lett. B 428, 105 (1998).

[5] E. Witten, Anti-de Sitter space and holography, Adv. Theor. Math. Phys. 2, 253 (1998).

[6] T. Banks, M. R. Douglas, G. T. Horowitz, and E. J. Martinec, AdS dynamics from conformal field theory, arXiv:hep-th/9808016.

[7] S. Terashima, AdS/CFT correspondence in operator formalism, J. High Energy Phys. 02 (2018) 019.

[8] V. Balasubramanian, P. Kraus, and A. E. Lawrence, Bulk versus boundary dynamics in anti-de Sitter space-time, Phys. Rev. D 59, 046003 (1999).

[9] I. Heemskerk, J. Penedones, J. Polchinski, and J. Sully, Holography from conformal field theory, J. High Energy Phys. 10 (2009) 079.

[10] A. L. Fitzpatrick and J. Kaplan, AdS field theory from conformal field theory, J. High Energy Phys. 02 (2013) 054.
[11] M. Miyaji, T. Numasawa, N. Shiba, T. Takayanagi, and K. Watanabe, Continuous Multiscale Entanglement Renormalization Ansatz as Holographic Surface-State Correspondence, Phys. Rev. Lett. 115, 171602 (2015).

[12] Y. Nakayama and H. Ooguri, Bulk locality and boundary creating operators, J. High Energy Phys. 10 (2015) 114.

[13] H. Verlinde, Poking holes in AdS/CFT: Bulk fields from boundary states, arXiv:1505.05069.

[14] I. Bena, On the construction of local fields in the bulk of $\operatorname{AdS}(5)$ and other spaces, Phys. Rev. D 62, 066007 (2000).

[15] A. Hamilton, D. N. Kabat, G. Lifschytz, and D. A. Lowe, Local bulk operators in AdS/CFT: A boundary view of horizons and locality, Phys. Rev. D 73, 086003 (2006).

[16] A. Hamilton, D. N. Kabat, G. Lifschytz, and D. A. Lowe, Holographic representation of local bulk operators, Phys. Rev. D 74, 066009 (2006).

[17] S. El-Showk and K. Papadodimas, Emergent spacetime and holographic CFTs, J. High Energy Phys. 10 (2012) 106.

[18] D. Kabat, G. Lifschytz, and D. A. Lowe, Constructing local bulk observables in interacting AdS/CFT, Phys. Rev. D 83, 106009 (2011).

[19] D. Kabat, G. Lifschytz, S. Roy, and D. Sarkar, Holographic representation of bulk fields with spin in AdS/CFT, Phys. Rev. D 86, 026004 (2012). 
[20] D. Kabat and G. Lifschytz, CFT representation of interacting bulk gauge fields in AdS, Phys. Rev. D 87, 086004 (2013).

[21] A. L. Fitzpatrick, J. Kaplan, and M. T. Walters, Universality of long-distance AdS physics from the CFT bootstrap, J. High Energy Phys. 08 (2014) 145.

[22] D. Kabat and G. Lifschytz, Bulk equations of motion from CFT correlators, J. High Energy Phys. 09 (2015) 059.

[23] D. Kabat and G. Lifschytz, Locality, bulk equations of motion and the conformal bootstrap, J. High Energy Phys. 10 (2016) 091.

[24] K. Goto, M. Miyaji, and T. Takayanagi, Causal evolutions of bulk local excitations from CFT, J. High Energy Phys. 09 (2016) 130.

[25] J. W. Kim, Explicit reconstruction of the entanglement wedge, J. High Energy Phys. 01 (2017) 131.

[26] K. Goto and T. Takayanagi, CFT descriptions of bulk local states in the AdS black holes, J. High Energy Phys. 10 (2017) 153.

[27] V. K. Dobrev, Intertwining operator realization of the AdS/CFT correspondence, Nucl. Phys. B553, 559 (1999).

[28] M. Duetsch and K. H. Rehren, Generalized free fields and the AdS-CFT correspondence, Ann. Inst. Henri Poincaré 4, 613 (2003).

[29] A. Almheiri, X. Dong, and D. Harlow, Bulk locality and quantum error correction in AdS/CFT, J. High Energy Phys. 04 (2015) 163.

[30] D. Harlow, TASI lectures on the emergence of bulk physics in AdS/CFT, Proc. Sci., TASI2017 (2018) 002 [arXiv: 1802.01040].

[31] A. C. Wall, Maximin surfaces, and the strong subadditivity of the covariant holographic entanglement entropy, Classical Quantum Gravity 31, 225007 (2014).

[32] M. Headrick, V.E. Hubeny, A. Lawrence, and M. Rangamani, Causality and holographic entanglement entropy, J. High Energy Phys. 12 (2014) 162.

[33] B. Czech, J. L. Karczmarek, F. Nogueira, and M. Van Raamsdonk, The gravity dual of a density matrix, Classical Quantum Gravity 29, 155009 (2012).

[34] D. L. Jafferis, A. Lewkowycz, J. Maldacena, and S. J. Suh, Relative entropy equals bulk relative entropy, J. High Energy Phys. 06 (2016) 004.
[35] R. Bousso, S. Leichenauer, and V. Rosenhaus, Light-sheets and AdS/CFT, Phys. Rev. D 86, 046009 (2012).

[36] S. Terashima, Classical limit of large $\mathrm{N}$ gauge theories with conformal symmetry, J. High Energy Phys. 02 (2020) 021.

[37] G. 't Hooft, On the quantum structure of a black hole, Nucl. Phys. B256, 727 (1985).

[38] N. Iizuka and S. Terashima, Brick walls for black holes in AdS/CFT, Nucl. Phys. B895, 1 (2015).

[39] J. D. Qualls, Lectures on conformal field theory, arXiv: 1511.04074.

[40] S. Rychkov, EPFL lectures on conformal field theory in $D \geq 3$ dimensions, arXiv:1601.05000.

[41] D. Simmons-Duffin, The conformal bootstrap, arXiv: 1602.07982.

[42] J. M. Maldacena and A. Strominger, AdS(3) black holes and a stringy exclusion principle, J. High Energy Phys. 12 (1998) 005.

[43] X.-X. Bai and Y. Zhao, A uniform asymptotic expansion for Jacobi polynomials via uniform treatment of Darboux's method, J. Approx. Theory 148, 1 (2007).

[44] H. Reeh and S. Schlieder, Bemerkungen zur unitäräquivalenz von lorentzinvarianten feldern, Nuovo Cimento 22, 1051 (1961).

[45] E. Witten, APS medal for exceptional achievement in research: Invited article on entanglement properties of quantum field theory, Rev. Mod. Phys. 90, 045003 (2018).

[46] P. Breitenlohner and D.Z. Freedman, Positive energy in anti-De Sitter backgrounds and gauged extended supergravity, Phys. Lett. 115B, 197 (1982).

[47] L. Chen and M. Ismail, On asymptotics of jacobi polynomials, SIAM J. Math. Anal. 22, 1442 (1991).

[48] Y. Suzuki, T. Takayanagi, and K. Umemoto, Entanglement Wedges from the Information Metric in Conformal Field Theories, Phys. Rev. Lett. 123, 221601 (2019); Y. Kusuki, Y. Suzuki, T. Takayanagi, and K. Umemoto, Looking at shadows of entanglement wedges, arXiv:1912.08423.

[49] C. Akers, S. Leichenauer, and A. Levine, Large breakdowns of entanglement wedge reconstruction, Phys. Rev. D 100, 126006 (2019).

[50] S. Ryu and T. Takayanagi, Holographic Derivation of Entanglement Entropy from AdS/CFT, Phys. Rev. Lett. 96, 181602 (2006); Aspects of holographic entanglement entropy, J. High Energy Phys. 08 (2006) 045. 